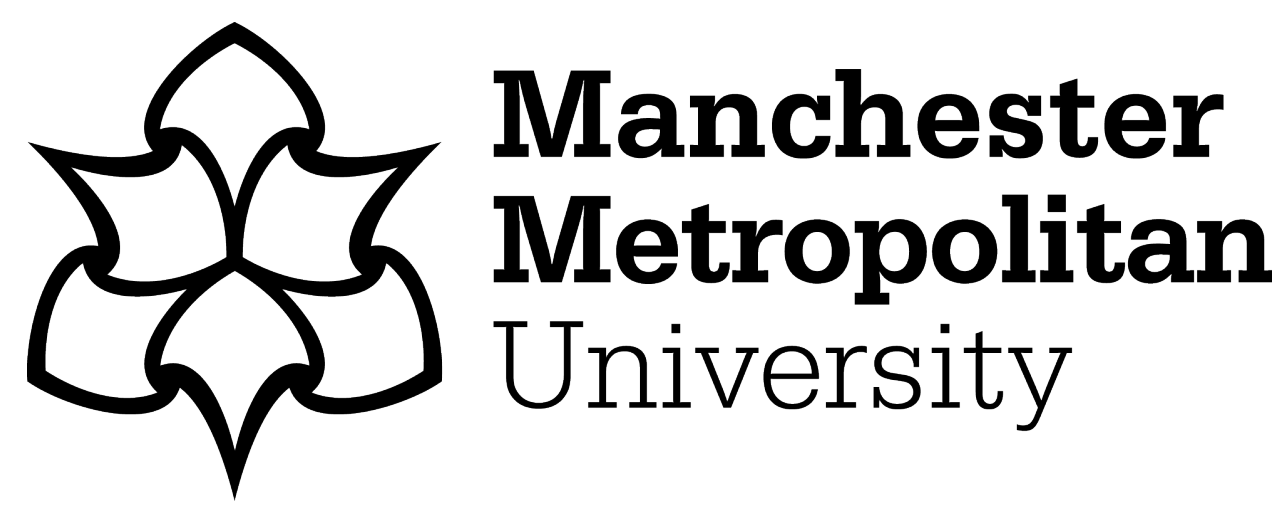

Brotons, A, Sanjuán, I, Foster, CW, Banks, CE, Vidal-Iglesias, FJ, SollaGullón, J and Iniesta, J (2016) A Facile and Cost-effective Electroanalytical Strategy for the Quantification of Deoxyguanosine and Deoxyadenosine in Oligonucleotides Using Screen-printed Graphite Electrodes. Electroanalysis, 28 (12). pp. 3066-3074. ISSN 1040-0397

Downloaded from: https://e-space.mmu.ac.uk/617898/

Version: Accepted Version

Publisher: Wiley

DOI: https://doi.org/10.1002/elan.201600272

Please cite the published version 


\title{
A facile and cost-effective electroanalytical strategy for the quantification of deoxyguanosine and deoxyadenosine in oligonucleotides using screen-printed graphite electrodes
}

\author{
Ariadna Brotons ${ }^{a}$, Ignacio Sanjuán $^{a}$, Christopher W. Foster ${ }^{c}$, Craig E. Banks $^{c}$, Francisco José Vidal-Iglesias $^{a}$, José \\ Solla-Gullón ${ }^{a}$ and Jesús Iniesta ${ }^{a, b^{*}}$ \\ a Institute of Electrochemistry, University of Alicante, 03690, San Vicente del Raspeig, Alicante, Spain \\ b Physical Chemistry Department, University of Alicante, 03690, San Vicente del Raspeig, Alicante, Spain \\ c Faculty of Science and Engineering, School of Science and the Environment, Division of Chemistry and Environmental Science, \\ Manchester Metropolitan University, Chester Street, Manchester M1 5GD, UK \\ * e-mail: jesus.iniesta@ua.es
}

Received: ((will be filled in by the editorial sttaff))

Accepted: ((will be filled in by the editorial sttaff))

\begin{abstract}
The development of electroanalytical methods for the detection and quantification of nucleotides in DNA offers vital implications in assessing the degree of oxidation or epigenetic modification in DNA. Unfortunately, the electrochemical response of oligonucleotides is strongly influenced by the size, composition and nucleic base sequence. In this article, an optimized analytical procedure for the enzymatically breakdown of the oligonucleotides to their corresponding nucleotides for the evaluation of the electrochemical response through the use of square wave voltammetry (SWV) is presented. Enzymatic digestion of oligonucleotides has been optimized in terms of buffer composition, digestion time, strategy for stopping the enzymatic reaction and filtration requirement for enzyme removal, and then compared to an established protocol. Under the optimized protocol SWV response of a number of untreated and enzymatically digested six-mer oligonucleotides, namely 5'-GGGGGG-3', 5'-AAAAAA-3', 5'-CGCGCG-3' and 5'-AAACGC-3' have been analysed, providing a higher sensitivity for the determination of guanosine and adenosine monophosphate species under digestion conditions with a more facile and cost effective procedure. The novel strategy for the enzymatically treated oligonucleotides in combination with the SWV response provides a proof of principle for feasible applications in the diagnosis of methylated guanosine in DNA as a potential biomarker due to its relation with cancer.
\end{abstract}

Keywords: Screen printed graphite electrode, enzymatic digestion, Square Wave Voltammetry, oligonucleotides.

DOI: 10.1002/elan.((will be filled in by the editorial sttaff))

\section{Introduction}

Nucleotides, nucleosides or nucleobases have been widely studied due to their important role within living organisms. Advances in the study of DNA are being focused on the investigation of molecular processes and the search for practical applications in different fields such as molecular diagnostics, food analysis, pathophysiological disorders, and disease diagnosis and treatment. Particularly, a number of research groups have paid considerable attention to the field of epigenetics which consists in the examination of the external or environmental factors that turn genes on and off, reflecting how cells 'read' them. Epigenetic modifications in DNA result in chemical alterations of nucleobases, without any change in the DNA sequence [1]. In recent years, the investigation of epigenetics has reached a significant importance as it has been linked to different human carcinomas [2], leukemia [3], lung [4], thyroid [4], pancreas [4] and prostate [5] tumours, amongst others. In this regard, a variety of analytical methods have emerged and developed for the determination of epigenetic modifications, such as bisulphite sequencing [6], 32Ppostlabeling [7] and chromatographic methods coupled to spectroscopic and spectrometric techniques [8] and electrochemical detection [9]. Even though these methods are sensitive, they are time-consuming and have high costs, which make them economically unfeasible for routine implementation. To overcome these drawbacks, novel methodological directions are aiming at the development of sensitive electrochemical sensors. These allow easy measurements, providing rapid and accurate results making them economically viable to be routinely implemented. Nevertheless, it is well-known that electrochemical devices do not display high reproducibility compared to those using separation via chromatographic techniques, and for that reason, extensive effort about the 
understanding of nucleic acid electrochemical behaviour is required. Although early works explored the reductive electrochemical behaviour of DNA and its related components [10], over recent years effort has been paid upon the direct oxidation of nucleobases, nucleosides and nucleotides. Many of these studies are based on the use of carbon materials, due to its higher reproducibility and sensitivity [11].

Carbon materials are widely employed due to their unique properties in terms of textural, structural and surface chemistry. Not only do these properties clearly affect the electrochemical response of nucleic base derivatives, but also the size and the physicochemical nature of the DNA moiety should be considered. In our previous work different 6-mer oligonucleotides, with the motif 5'XXXCGC-3', were used for the investigation of the anodic oxidation at a screen-printed graphite electrode via square wave voltammetry and then compared with the same equivalent concentration of cytosine (C) [12]. Such results exhibited distinct anodic peak intensities for $\mathrm{C}$ electrooxidation as a function of the oligonucleotide sequence XXX for the same equivalent concentration of the nucleobase C. Data obtained by Brotons et al. [12] is consistent with those displayed by Pumera's group where they stated that the electro-oxidation of oligonucleotides is influenced by their position and neighbouring nucleobases [13]. Such electrochemical response was attributed to the steric effect caused by electrostatic interactions between the positively charged electrode surface and the negatively charged phosphate group. Moreover, the electrode interaction between the oligonucleotide and the electrode can cause reorganization of the oligonucleotide at the surface after adsorption and prior to charge transfer [14]. Furthermore, large oligonucleotides generally exhibit low electrochemical sensitivity resulting from conformational problems triggered, on the one hand, by the increased distance between each nucleobase and the electrode surface, and on the other hand, because the diffusion coefficient decreases with increasing DNA residues [12, 15]. In an attempt to solve or minimise the above issue, Kato's group used a methodology for the determination of methylation grade [16] based on the enzymatic hydrolysis of oligonucleotides by comparing the SWV response of digested methylated and non-methylated 20-mer and 60mer oligonucleotides, respectively, with a $\mathrm{CpG}$ motif in order to reach free deoxyguanine monophosphate (dGMP), deoxycytosine monophosphate (dCMP) and methyldeoxycytosine monophosphate (mdCMP) oligonucleosides, avoiding surface fouling and improving the electrochemical performance of dGMP, dCMP and mdCMP. However, the procedure used by Kato's group suffers from a lack of electroanalysis immediacy, since the digestion protocol lasts more than $120 \mathrm{~min}$, besides being expensive due to the need of spin columns to remove the enzyme. Even though this group optimised the digestion time for $\mathrm{CpG}$ oligonucleotides in the absence and presence of methylcytosine with formidable electrochemical response on the nanocarbon film electrodes, they have not explored the electrochemical response of digested oligonucleotides in the presence of the enzyme, the presence of adenine or thymine in the oligonucleotide and their effect on the enzyme reactivity and, finally, the feasibility of using other enzymes.

In this work we detail a facile and low cost methodology following a similar standardised enzymatic digestion process of a number of model oligonucleotides using disposable screen-printed graphite electrodes (SPGE), which are easily manufactured and low cost mass produced compared with the use of more expensive materials such as boron doped diamond or nanocarbon films. Since each SPGE has only one use, problems such as electrode fouling are avoided. In this paper, the electrochemical response of 6-mer oligonucleotides and the nucleosides monophosphate obtained following an enzymatic hydrolysis have been investigated using the Nuclease S1 enzyme through SWV in acetic/acetate buffer solution $\mathrm{pH}$ 5.0. Accordingly, as a proof of concept, several types of 6-mer oligonucleotides consisting of poly(A): 5'-AAAAAA-3', poly(G): 5'-GGGGGG-3', poly $(\mathrm{C})$ : 5'-CCCCCC-3', 5'-CGCGCG-3' and 5'AAACGC-3', have been explored at the SPGE platforms at different concentrations. The relevance of using 5'CGCGCG-3' oligonucleotide lies in the similarity with $\mathrm{CpG}$ islands, which are cytosine-guanine rich regions located within the promoter region of genes, and exhibit an important function in gene regulation [1]. Enzymatic digestion time, temperature, buffer enzymatic composition, the need of removing the enzyme from the enzymatic solution before electrochemical measurements and pretreatment of the SPGE have been explored to get a feasible methodology for the improvement of the electroanalytical determination of free nucleotides monophosphate in DNA.

\section{2-Material and methods}

\subsection{Materials and chemicals}

Deoxyadenosine monophosphate (dAMP), deoxyguanosine monophosphate (dGMP) and deoxycytidine monophosphate (dCMP) were obtained from Sigma Aldrich. 6-mer oligonucleotide of A (poly $(\mathrm{A})$ : 5'-AAAAAA-3'), 6-mer oligonucleotide of $\mathrm{G}$ (poly $(\mathrm{G})$ : 5'-GGGGGG-3'), 6-mer oligonucleotide of C (poly(C): 5'CCCCCC-3') and oligonucleotides 5'-CGCGCG-3' and 5'-AAACGC-3' were obtained at a micromolar scale, purified by HPLC (from Fisher Scientific) and used as received without any further purification. Ultrapure water (Milli-Q 18.2 M $\Omega \mathrm{cm}$ ) was used for all solutions. 0.1 M 
acetate buffer solutions (sodium acetate, Scharlau Chemie S.A., reagent grade) were prepared by setting $\mathrm{pH}$ values with glacial acetic acid (J. T. Backer, 99-100\% purity) and concentrated $\mathrm{NaOH}$ (Scharlau Chemie S.A., reagent grade) solutions. Measurements of $\mathrm{pH}$ were carried out with a Crison Micro pH 2000 pH-meter. We have also used the commercial buffer recommended for the enzymatic hydrolysis consisting of $200 \mathrm{mM}$ sodium acetate (pH 4.5), $1.5 \mathrm{M} \mathrm{NaCl}$ and $10 \mathrm{mM} \mathrm{ZnSO}_{4}$ ).

The enzyme Nuclease S1 from "Aspergillus oryzae" (100 Units $\mu \mathrm{L}$ ) dissolved in a storage buffer solution was purchased from Fisher Scientific. This storage buffer consisted of a solution with $20 \mathrm{mM}$ Trizma hydrochloride $(\mathrm{pH} 7.5), 50 \mathrm{mM} \mathrm{NaCl}, 0.1 \mathrm{mM} \mathrm{ZnCl} 2$ and $50 \%(\mathrm{v} / \mathrm{v})$ glycerol. When necessary, digested oligonucleotide solutions were filtered using a spin column (Amicon Ultra $0.5 \mathrm{~mL}$ centrifugal filters from EMD Millipore). Nuclease $\mathrm{S} 1$ and oligonucleotide solutions were stored at $-20{ }^{\circ} \mathrm{C}$ before use.
2.2. Fabrication of carbon based screen-printed electrodes

Screen-printed graphite electrodes (SPGE) were fabricated in-house with appropriate stencil designs using a microDEK 1760RS screen-printing machine (DEK, Weymouth, UK). A carbon-graphite ink formulation was previously used [17] and was screen-printed onto a polyester flexible film (Autostat, 250 micron thickness). This layer was cured in a fan oven at $60{ }^{\circ} \mathrm{C}$ for 30 minutes. Next, a silver/silver chloride reference electrode was included by screen-printing $\mathrm{AgCl} / \mathrm{Ag}$ paste (Gwent Electronic Materials Ltd, UK) onto the plastic substrate. Lastly a dielectric paste ink (Gwent Electronic Materials Ltd, UK) was printed to cover the connections and define the $3 \mathrm{~mm}$ diameter graphite working electrode (projected geometric area of $0.071 \mathrm{~cm}^{2}$ ). After curing at $60{ }^{\circ} \mathrm{C}$ for 30 minutes, the screen-printed electrode was ready to use. Similar SPGE platforms have been electrochemically characterized in a previous contribution [18], where the electroactive area was determined to be $0.052 \mathrm{~cm}^{2}$ with a coefficient of variation of $6.22 \%(\mathrm{~N}=6)$.

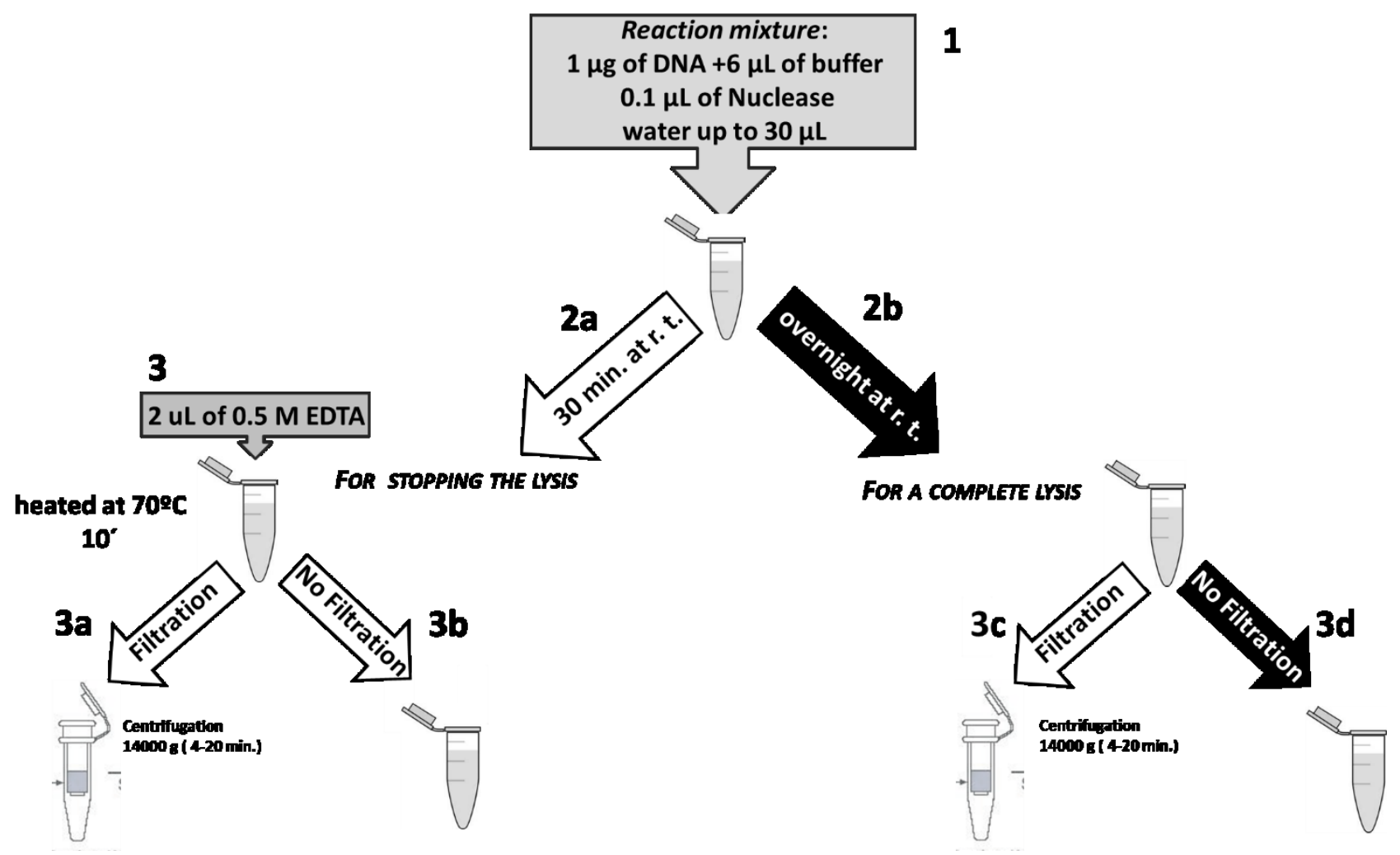

Fig 1. Scheme describing different procedure steps for the oligonucleotide enzymatic digestion and sample preparation based on the use of the trade protocol: step 1) Reaction mixture preparation (1 $\mu \mathrm{g}$ of DNA $+6 \mu \mathrm{L}$ of commercial buffer $+0.1 \mu \mathrm{L}$ of Nuclease S1 + water up to $30 \mu \mathrm{L}$ for the enzymatic digestion of a DNA sample); step 2a) Incubation for 30 min. at room temperature; step 2b) Incubation overnight at room temperature; step 3) Addition of EDTA for stopping the enzymatic digestion; steps 3a-c) sample filtration; steps 4b-d) no sample filtration. Black arrows highlight the optimum procedure followed in this work. 


\subsection{Electrochemical measurements.}

SWV measurements were carried out using a $\mu$ Autolab III potentiostat/galvanostat (Metrohm Autolab, The Netherlands) and controlled by GPES software version 4.9 for Windows XP. All experiments were performed at $22 \pm 2{ }^{\circ} \mathrm{C}$.

Unless stated elsewhere, SWV parameters are the following: modulation amplitude, $50 \mathrm{mV}$; modulation frequency $8 \mathrm{~Hz}$; modulation step $(\Delta \mathrm{E}), 1 \mathrm{mV}$. All potentials are referred to a $\mathrm{AgCl} / \mathrm{Ag}$ pseudo-reference electrode. Prior to the electrochemical measurement, a conditioning pretreatment of the graphitic working electrodes was performed, in which the electrode was submitted to five consecutive cyclic voltammetry cycles between 0 and $1.3 \mathrm{~V}$ vs $\mathrm{AgCl} / \mathrm{Ag}$ at $50 \mathrm{mV} \mathrm{s}^{-1}$ in $0.1 \mathrm{M}$ acetate buffer $\mathrm{pH}$ 5.0. Thereafter the SPGE platform was thoroughly rinsed with acetate buffer solution before the electrochemical measurement. Generally, $50 \mu \mathrm{L}$ of the solutions containing a certain amount of untreated and enzymatically treated oligonucleotides of study were placed onto the screen printed electrode and held at $0 \mathrm{~V}$ for 5 seconds before starting the SWV measurements. Limits of detection (LoD) and quantification (LoQ) were calculated as three and ten times the noise level, respectively.

\section{Results and discussion}

\subsection{Optimization of the experimental conditions for the enzymatic digestion of oligonucleotides}

A number of experimental variables have been evaluated in order to optimise the standard protocol for the enzymatic digestion of oligonucleotides to nucleosides monophosphate. Nuclease S1 degrades single-stranded nucleic acids by cleavage of the phosphodiester bond to release 5'-phosphorylated products (mononucleotides or short oligonucleotides) [19].

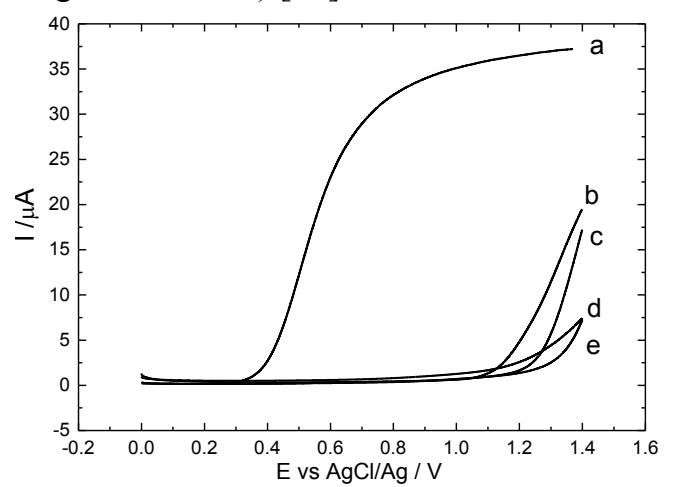

Fig 2. SWV responses for interferents present in the sampling buffer after the oligonucleotide digestion procedure: a) $30 \mathrm{mM}$ EDTA in $0.1 \mathrm{M}$ acetate buffer $\mathrm{pH} 5.0$, b) $300 \mathrm{mM} \mathrm{NaCl}$, c) 0.1 $\mathrm{M}$ acetate buffer solution $\mathrm{pH} 5.0$, d) $2 \mathrm{mM} \mathrm{ZnSO}_{4}$ and e) 0.33
Units of the enzyme Nuclease S1 solution in $0.1 \mathrm{M}$ acetate buffer solution $\mathrm{pH}$ 5.0.

Figure 1 depicts the different protocols used for the enzymatic digestion of oligonucleotides in order to optimise the procedure and minimise the effect of the interferences present within the final sample when applied the standardised commercial protocol. The reaction mixture is customarily prepared by adding for each $\mu \mathrm{g}$ of DNA, $6 \mu \mathrm{L}$ of buffer, $0.1 \mu \mathrm{L}$ of Nuclease S1 and Milli-Q water up to $30 \mu \mathrm{L}$, as shown in step 1 of Figure 1. Alternatively, $0.1 \mathrm{M}$ acetate buffer solution $\mathrm{pH}$ 5.0 was used instead of the commercial buffer solution. All sample dilutions were performed using $0.1 \mathrm{M}$ acetate buffer solution $\mathrm{pH}$ 5.0.

According to the specifications of the standard protocol and after the incubation of the enzymatic reaction mixture for $30 \mathrm{~min}$ at room temperature (step 2a in figure 1), enzyme inhibition has to be performed by adding $2 \mu \mathrm{L}$ of $0.5 \mathrm{M}$ EDTA solution into the reaction mixture for the complexation of free $\mathrm{Zn}$ (II) ions [20], as depicted in step 3 in Figure 1; subsequently the reaction mixture is heated up to $70^{\circ} \mathrm{C}$ for 10 minutes. After inhibiting the enzymatic digestion the final reaction can be also subjected to filtration, as shown in step 3a in figure 1, to remove the Nuclease S1 or simply the reaction mixture is ready to be explored electrochemically using the SPGE, as described in step $3 \mathrm{~b}$ in figure 1 . It is worth noting that the final reaction mixture is a complex fluid made of a wide number of organic and inorganic compounds, which are required to be explored to assess whether or not they affect the electrochemical response of DNA related components. In that respect, Kato et al. highlighted the need of the removal of the enzyme endonuclease P1 using spin cut-off centrifuge tubes before analysing the electrochemical SWV response of dGMP and dCMP on nanocarbon electrodes through electron cyclotron resonance (ECR) [16]. Even though they efficiently removed the endonuclease P1 enzyme from the final solution they ignored the possible effect of the presence of the enzyme into the buffer upon the electrochemical response with the aim of discarding the step 3a in Figue 1. Furthermore, for a more practical approach, the use of SPGE devices instead ECR carbon film electrodes displays several advantages in terms of facile and cost effective fabrication.

Fig 2 shows the SWV responses of all inherent interferents found during the lysis procedures of the oligonucleotides, such as $\mathrm{NaCl}$ (as mentioned above and present in the commercial buffer), $\mathrm{ZnSO}_{4}$ (a cofactor needed for the correct activity of the enzyme which is present in the commercial buffer), Nuclease $\mathrm{S} 1$ (which is a single-strand-specific endonuclease that hydrolyses single-stranded RNA or DNA into 5' mononucleotides), and EDTA (used to stop the hydrolysis reaction). It is 
worth noting that only the electro-oxidation of EDTA starts at less positive potentials, near $+0.4 \mathrm{~V}$. Accordingly, the stronger electro-oxidative current observed during the SWV response utterly interferes with the SWV response of either the nucleotide dGMP and dAMP, as demonstrated in the electronic supplementary information, Figure ESI-1. Even though the addition of a certain amount of EDTA solution is only required for ceasing the enzyme activity by complexing the $\mathrm{Zn}^{2+}$ cofactor, in the light of the results, the use of EDTA solution was discarded and the enzymatic digestion step was extended overnight, as depicted in step 2b in Figure 1. In terms of the electrochemical SWV response, no significant differences were obtained when comparing the use of the trade buffer solution at $\mathrm{pH} 4.5$ (vide supra) and our acetate buffer solution at pH 5.0 (results not shown). Hence, the commercial buffer solution was substituted by $0.1 \mathrm{M}$ acetate buffer solution $\mathrm{pH}$ 5.0.

Sample filtration is a common procedure for assessing the electrochemical response after enzymatic digestion in order to remove completely the Nuclease S1 enzyme, as recently was proposed by Kato's group [16]. The presence of macromolecules can cause a detrimental effect in terms of fouling of the electrochemically active surface area and therefore create a loss of the SWV response. However, even though the removal of the Nuclease S1 might be beneficial for the improvement of the SWV response (of the digested oligonucleotides), cleaning or pretreatment of filter devices is required before using and therefore creates a time-consuming technique. Moreover, the use of filters based upon the polymeric matrix can produce undesirable adsorption of organic molecules thus significantly reducing the amount of analyte. Nevertheless, a comparative SWV response study of both the filtered and non-filtered samples regarding steps $3 \mathrm{c}$ and $3 \mathrm{~d}$ (from the protocol described in Figure 1), respectively, is depicted in Figure 3, where the peak intensity of the enzymatic digested polyG oligonucleotide decreases when the sample passes through the filter, possibly due to adsorption phenomena, thus reducing the final concentration of sample to be measured. Adsorption of dAMP was also demonstrated when polyA oligonucleotide was digested under the same experimental conditions (results not shown). Since Nuclease $\mathrm{S} 1$ is electrochemically inactive within a potential window from 0 to $1.0 \mathrm{~V}$, according to the results shown in Figure 2, filtration of the digested sample was discarded, thereby reducing the analysis time as well as avoiding uncontrolled adsorption of different analytes on the filter device.

Figure 4 shows the effect of the thermal treatment used to stop the enzymatic digestion on the SWV response of a $18 \mu \mathrm{M}$ digested polyG solution. Established protocols indicate that the addition of an EDTA solution together with the heating of the sample solution is recommended to cease the lytic activity, as described in step 3 in figure 1. Figure $4 \mathrm{~A}$ shows the SWV response for the electrooxidation of polyG oligonucleotide after digestion and subsequent thermal pretreatment at $70{ }^{\circ} \mathrm{C}$ during 10 minutes. Such SWV reveals an anodic peak at $+0.55 \mathrm{~V}$ prior to the main electro-oxidation of the dGMP, at a peak potential of $+0.79 \mathrm{~V}$. The first and smaller peak is, in part, due to partially thermal decomposition of either the enzyme or the oligonucleotide due to the high temperature. The potential and current peaks associated with the dGMP oxidation are similar when the enzymatic digestion takes place overnight at $25^{\circ} \mathrm{C}$, as shown in Figure $4 \mathrm{~B}$. Interestingly, the peak potential at $+0.55 \mathrm{~V}$ is not present under these softer conditions. Again, even though the use of thermal treatment is appropriate for stopping the enzymatic reaction, the addition of EDTA still precludes the use of step 3 mainly due to the high interference of EDTA. On the contrary, the use of a more extended digestion, though more time consuming, leads to a complete digestion of the oligonucleotide under softer conditions.

Summarising, the assessment of variables involved during the enzymatic breaking down of the oligonucleotides to the corresponding nucleotides such as enzymatic digestion time, addition of EDTA or thermal pretreatment, and the convenience of using filtration has conducted to an optimised protocol in terms of cost effective, low interference effect and easy-handling. This optimised protocol is highlighted in Figure 1 by black arrows and white text.

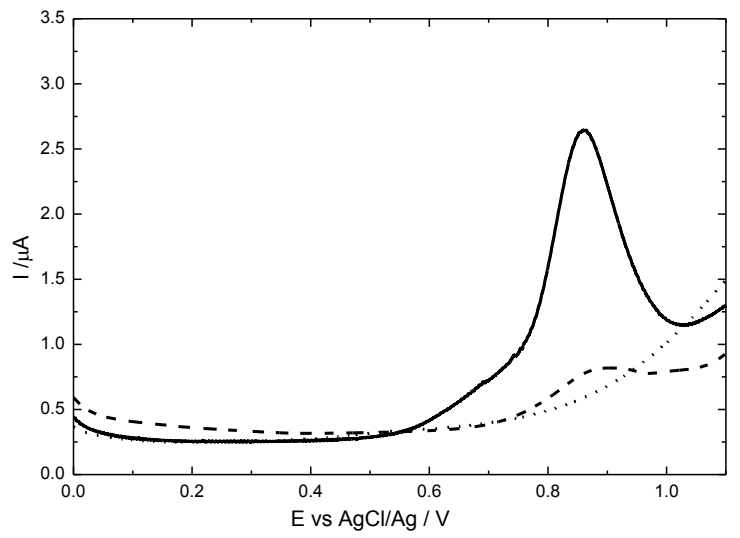

Fig 3. SWV responses of $7.5 \mu \mathrm{M}$ of digested and filtered polyG solution with an equivalent concentration of $45 \mu \mathrm{M}$ dGMP (dashed line) and digested $7.5 \mu \mathrm{M}$ polyG without filtration (solid line) at SPGE. SWV response of $0.1 \mathrm{M}$ acetate buffer $\mathrm{pH}$ 5.0 (dotted line). 

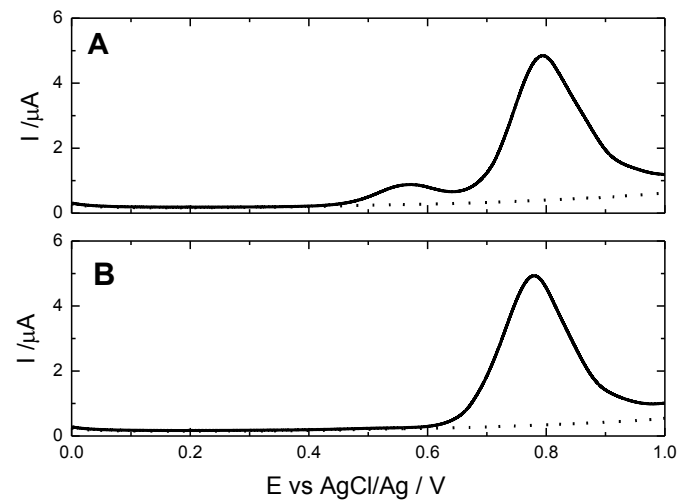

Figure 4. SWV response for $18 \mu \mathrm{M}$ digested polyG solution (solid line) under different experimental conditions increasing the temperature up to $70{ }^{\circ} \mathrm{C}$ for 10 minutes after oligonucleotide digestion (figure $\mathrm{A}$ ) and with no thermal pretreatment after oligonucleotide digestion (figure B). $0.1 \mathrm{M}$ acetate buffer $\mathrm{pH}$ 5.0 .

\subsection{SWV behaviour of digested polyA and polyG, under optimal conditions.}

Figure 5A shows the SWV response of the oxidation of both the polyG after the enzymatic digestion (solid line) following the protocol highlighted by black arrows (Figure 1) and that prior to the enzymatic digestion of polyG (dotted line). The peak intensity of the oxidation of the untreated polyG $(50 \mu \mathrm{M}$ of oligonucleotide which corresponds to an equivalent concentration of $300 \mu \mathrm{M}$ dGMP) is $0.37 \mu \mathrm{A}$ with a peak potential of $+0.83 \mathrm{~V}$. In the case of the enzymatically digested polyG, a current peak of $4.9 \mu \mathrm{A}$ and a peak potential of $+0.86 \mathrm{~V}$ is obtained (with $18 \mu \mathrm{M}$ of oligonucleotide and an equivalent concentration of $108 \mu \mathrm{M}$ of dGMP). It is worthy to observe the improvement of treated oligonucleotide ratio in the SWV response with a peak current to concentration of $\sim 40$. Moreover, peak potentials remained nearly unaltered regardless of the concentration and treatment of the oligonucleotide.

Figure 5B depicts the SWV response for the electrooxidation of the oligonucleotide polyA, with and without the enzymatic digestion. An anodic peak at $+1.18 \mathrm{~V}$ attributed to the oxidation of polyA is observed with a current peak of $5.91 \mu \mathrm{A}$ with $300 \mu \mathrm{M}$ untreated polyA (with an equivalent concentration of $1800 \mu \mathrm{M}$ dAMP) compared to a current peak of $9.4 \mu \mathrm{A}$ with a concentration of $18 \mu \mathrm{M}$ treated polyA $(108 \mu \mathrm{M}$ dAMP as equivalent concentration). The current peak to concentration of the enzymatic digested polyA ratio is close to 25 . The observed improvement in the sensitivity detection of both polyG and polyA can be attributed to a shorter average distance between each electroactive base and the surface of the electrode, giving rise to less conformational impediments together with a diminution of the diffusion coefficient because of the molecular size $[12,21]$.

Figures $6 \mathrm{~A}$ and $6 \mathrm{~B}$ show the electro-oxidation responses of different concentrations of dGMP (range 10-106 $\mu \mathrm{M}$ dGMP) and enzymatically treated polyG (with a concentration range between 15.6-72 $\mu \mathrm{M}$ dGMP) respectively, in a $0.1 \mathrm{M}$ acetate buffer solution ( $\mathrm{pH} 5.0$ ). Inset figures display the calibration curves of current versus concentration of the treated polyG after background subtraction (Figure 6B) and free dGMP (Figure 6A). In both cases a linear relationship was obtained with the following equations: $\operatorname{Ip} /(\mu \mathrm{A})=0.042 \pm$ $0.004\left(\mu \mathrm{A} \mu \mathrm{M}^{-1}\right)+0.3 \pm 0.1(\mu \mathrm{A})\left(\mathrm{R}^{2}=0.996\right)$ for the free $\mathrm{dGMP}$ and $\mathrm{Ip} /(\mu \mathrm{A})=0.034 \pm 0.002\left(\mu \mathrm{A} \mu \mathrm{M}^{-1}\right)+0.41 \pm$ $0.072(\mu \mathrm{A})\left(\mathrm{R}^{2}=0.999\right)$ for the digested polyG, for experiments performed in triplicate. For the free dGMP we obtained a limit of detection of $7.97 \mu \mathrm{M}$ and a limit of quantification of $26.58 \mu \mathrm{M}$ and a sensitivity of $0.042 \mu \mathrm{A}$ $\mu \mathrm{M}^{-1}$. In the case of the polyG after lysis, we obtained a limit of detection of $2.42 \mu \mathrm{M}$ and a limit of quantification of $8.1 \mu \mathrm{M}$, and a sensibility of $0.034 \mu \mathrm{A} \mu \mathrm{M}^{-1}$.

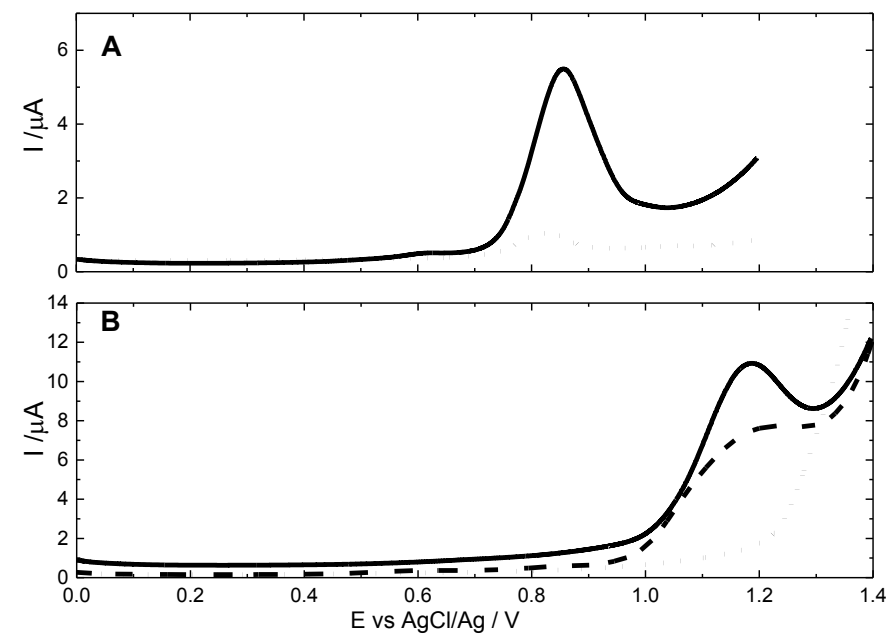

Fig. 5. (A) SWV response of $50 \mu \mathrm{M}$ polyG (dotted line) and 18 $\mu \mathrm{M}$ dGMP after enzymatic digestion of a six-mer polyG (solid line) in $0.1 \mathrm{M}$ acetate buffer $\mathrm{pH}$ 5.0. (B) SWV response of 300 $\mu \mathrm{M}$ polyA (dashed line) and $18 \mu \mathrm{M}$ dAMP after enzymatic digestion of a six-mer polyA (solid line) in $0.1 \mathrm{M}$ acetate buffer pH 5.0 (dotted line).

Figures $7 \mathrm{~A}$ and $7 \mathrm{~B}$ show the SWV responses for the electro-oxidation of different dAMP concentrations (with a range concentration between 20-108 $\mu \mathrm{M}$ dAMP) and enzymatically digested polyA within a range concentration of 16-108 $\mu \mathrm{M}$ dAMP) respectively in a 0.1 $\mathrm{M}$ acetate buffer solution $\mathrm{pH}$ 5.0. Inset figures display the calibration curves of current versus concentration of free dAMP (Figure 7A) and enzymatically digested polyA (Figure 7B), proving a linear correlation with the equation $\mathrm{Ip} /(\mu \mathrm{A})=0.051 \pm 0.005\left(\mu \mathrm{A} \mu \mathrm{M}^{-1}\right)+1.8 \pm 0.3$ 
$(\mu \mathrm{A})\left(\mathrm{R}^{2}=0.995\right)$ for the free dAMP and the equation $\mathrm{Ip} /(\mu \mathrm{A})=0.057 \pm 0.005\left(\mu \mathrm{A} \mu \mathrm{M}^{-1}\right)+1.3 \pm 0.3(\mu \mathrm{A})$ $\left(\mathrm{R}^{2}=0.996\right)$ for enzymatically digested polyA. For the free dAMP calibration curve we obtained a limit of detection of $8.08 \mu \mathrm{M}$ and a limit of quantification of 27 $\mu \mathrm{M}$ and a sensitivity of $0.051 \mu \mathrm{A} \mu \mathrm{M}^{-1}$. In the case of the polyA after enzymatic digestion we obtained a limit of detection of $8.1 \mu \mathrm{M}$ and a limit of quantification of 27

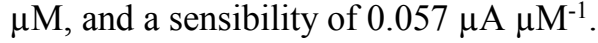
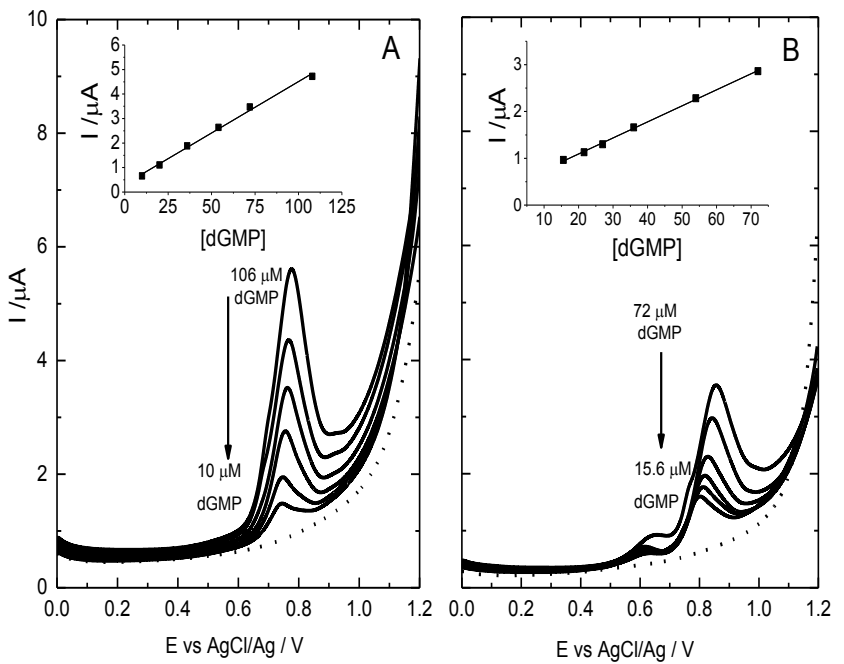

Fig. 6. SWV responses free dGMP (A) in $0.1 \mathrm{M}$ acetate buffer pH 5.0 with different concentrations $(10,20,36,54,72,106$ $\mu \mathrm{M}$ ) and for the enzymatically digested polyG (B) in $0.1 \mathrm{M}$ acetate buffer solution $\mathrm{pH} 5.0$ with different final dGMP concentrations $(15.6,21.6,27,36,54,72 \mu \mathrm{M})$. Background currents are plotted with dashed lines. Inset figures depict the calibration curves of current intensity versus dGMP concentrations for each case.
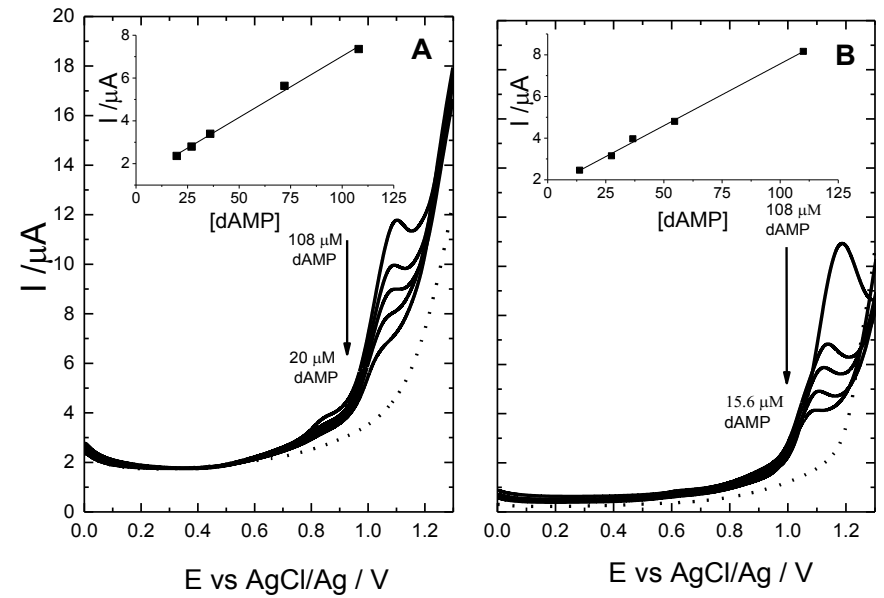

Fig. 7. SWV responses of free dAMP $(20,36,54,72,108 \mu \mathrm{M})$ (A) and polyA digested $(15.6,27,36,54,72,108 \mu \mathrm{M})(\mathrm{B})$ in $0.1 \mathrm{M}$ acetate buffer solution ( $\mathrm{pH}$ 5.0). Background currents are plotted with dashed lines. Inset figures depict the calibration curves of current intensity with dAMP and enzymatically treated polyA concentration for the linear range.

Summarising, both SWV responses depicted in Figures 6 and 7 comparing to the electro-oxidation of enzymatically treated polyG and polyA six-mers with the electrochemical SWV response of free dGMP or dAMP lead to an excellent match in terms of peak potential and currents. Such results demonstrate that the enzymatic protocol established in Figure 1 by black arrows is simple and effective for the sensing of dGMP and dAMP within DNA.

\subsection{SWV behaviour of enzymatically digested $5^{\prime}$ -

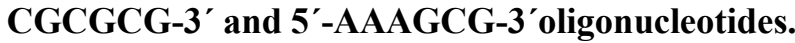

Next, we turn to the exploration of the SWV of a more complicated oligonucleotide, which simulates a short fragment of $\mathrm{CpG}$ island (5'-CGCGCG-3'). Figure 8 depicts the electrochemical response of the enzymatically treated 5'-CGCGCG-3', where the presence of the dGMP provides an electrochemical oxidation peak at a potential range of $0.78-0.87 \mathrm{~V}$. Moreover, Figure 8 reveals linear behaviour of the peak current obtained (from the electrooxidation of dGMP from the treated 5'-CGCGCG-3') with concentration (varying between 2.6-18 $\mu \mathrm{M}$ 5'CGCGCG-3', i.e. between 7.8-54 $\mu \mathrm{M}$ dGMP) in a $0.1 \mathrm{M}$ acetate buffer solution $\mathrm{pH}$ 5.0. Figure 8 (inset) depicts a linear correlation between the calibration curve of current intensity versus concentration of the enzymatically treated oligonucleotide $5^{\prime}$-CGCGCG-3' fitted by the following equation: $\mathrm{Ip} /(\mu \mathrm{A})=0.034 \pm 0.002\left(\mu \mathrm{A} \mu \mathrm{M}^{-1}\right)+$ $0.49 \pm 0.07(\mu \mathrm{A})\left(\mathrm{R}^{2}=0.996\right)$. Limit of detection and limit of quantification were $4.96 \mu \mathrm{M}$ and $16.55 \mu \mathrm{M}$ respectively and a sensitivity of $0.034 \mu \mathrm{A} \mu \mathrm{M}^{-1}$ which is

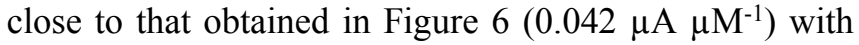
enzymatically treated six-mer polyG.

Figure 8 also reveals that the electro-oxidation of the deoxycytidine nucleoside monophosphate (dCMP), is not taking place under our experimental conditions or otherwise it occurs close to the electro-oxidation of the background. Such response is similar to that observed when exploring the electro-oxidation of the untreated polyC in $0.1 \mathrm{M}$ acetate buffer $\mathrm{pH} 5.0$ solutions, where an anodic peak is observed at potentials close to $+1.1 \mathrm{~V}$, ascribed to the oxidation of dCMP within the oligonucleotide as shown in Figure ESI-2. However, this signal is not observed after the digestion of the oligonucleotide. Similarly, Figure ESI-3A compares the SWV response of the untreated and enzymatic digested oligonucleotide 5'-CGCGCG-3' in which less positive anodic peak potentials are observed at ca. +0.9 and +1.2 $\mathrm{V}$ for the untreated oligonucleotide where the neighbouring effect of dGMP leads to a positive shift of 
$100 \mathrm{mV}$ for the anodic oxidation of dCMP. On the contrary, only an anodic peak at about $0.9 \mathrm{~V}$ for the oxidation of dGMP after digested treatment was observed. Such SWV behaviour can be exclusively attributed to the nature of the carbonaceous electrode. Once again, the evaluation of peak current intensity for a certain equivalent concentration of dGMP of Figure 8 (inset) is half to that value obtained from inset figure of figure $6 \mathrm{~B}$.

Finally, we have explored the SWV response of the enzymatically treated oligonucleotide 5'-AAAGCG-3', obtaining two different peaks centred at approximately $0.82-0.85 \mathrm{~V}$ and $1.11-1.15 \mathrm{~V}$, which are ascribed to the electro-oxidation of dGMP and dAMP, respectively, as shown in Figure 9; however, again no current peak was clearly observed for the electro-oxidation of dCMP. Figure 9 also displays the influence of the concentration of enzymatically treated $5^{\prime}$-AAAGCG-3' on the SWV response within a concentration range of 18-37 $\mu \mathrm{M}$ dAMP and $12-24 \mu \mathrm{M}$ dGMP in a $0.1 \mathrm{M}$ acetate buffer $\mathrm{pH}$ 5.0 solution. Figure 9 (inset) displays the calibration curves of free dGMP and dAMP after digestion of the oligonucleotide. The calibration curves obtained were $\mathrm{Ip} /(\mu \mathrm{A})=0.06 \quad \pm 0.01\left(\mu \mathrm{A} \quad \mu \mathrm{M}^{-1}\right) \quad-0.2 \quad \pm 0.2(\mu \mathrm{A})$ $\left(\mathrm{R}^{2}=0.989\right)$ for the nucleotide $\mathrm{dGMP}$ and $\mathrm{Ip} /(\mu \mathrm{A})=0.09 \pm$ $0.02\left(\mu \mathrm{A} \mu \mathrm{M}^{-1}\right)-0.2 \pm 0.7(\mu \mathrm{A})\left(\mathrm{R}^{2}=0.969\right)$ for the nucleotide dAMP. The limit of detection for dGMP was $3.13 \mu \mathrm{M}$ with a limit of quantification of $10.44 \mu \mathrm{M}$, with

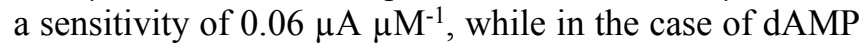
a limit of detection of $7.2 \mu \mathrm{M}$ and limit of quantification of $24 \mu \mathrm{M}$ was obtained, with a sensitivity of $0.09 \mu \mathrm{A} \mathrm{\mu \textrm {M } ^ { - }}$ ${ }^{1}$. A proper comparison with the peak current correlation with free dAMP (Figure 7) concentration could not be obtained mainly due to the simultaneous presence of three nucleotides.

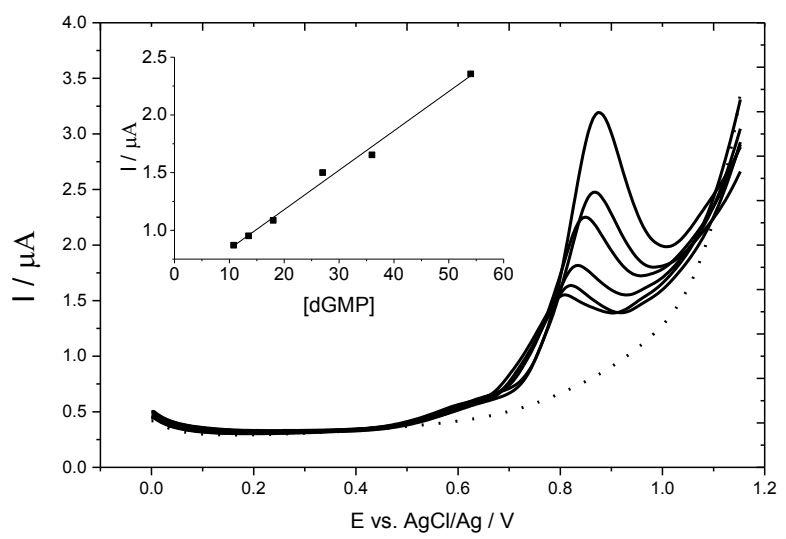

Fig. 8. SWV response of enzymatically treated 5' -CGCGCG-3' with concentration. Inset figure depicts the calibration curve of current intensity with dGMP concentration for the linear range. Background signal ( $0.1 \mathrm{M}$ acetate buffer $\mathrm{pH} 5.0$ solution) is plotted with a dashed line.

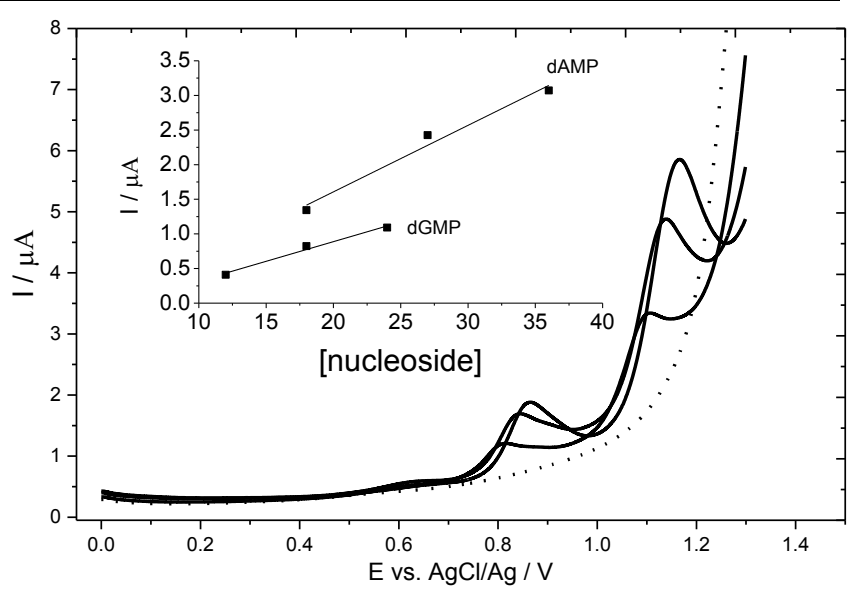

Fig. 9. SWV response of enzymatically treated 5'-AAACGC-3 with different concentrations. Background currents are plotted with dashed lines (0.1 M acetate buffer $\mathrm{pH}$ 5.0). Inset figure depicts the calibration curve of current intensity with dAMP and dGMP concentration for the linear range $(\mathrm{dAMP}=18,27$, $37 \mu \mathrm{M} ; \mathrm{dGMP}=12,18,24 \mu \mathrm{M})$.

Summarising, each type of nucleotide can provide a characteristic SWV response with quantitative information about the composition of the oligonucleotide after its enzymatic digestion. Particularly, the assessment of the SWV responses of different oligonucleotides based on $\mathrm{CpG}$ islands in genes is of vital importance for the diagnosis of methylation degree. Our developed procedure for the enzymatic digestion of oligonucleotides could unambiguously identify the presence of methylated dGMP. Our previous works performed by Brotons et al. [22] and Sanjuán et al. [23] have demonstrated that the SWV responses of N7-methylguanine and guanine may be distinguished at SPGE platforms and boron doped diamond electrodes, with no interference effect when adenine nucleic base is present. Future work needs to be envisaged for the examination of the electrochemical response of more complex oligonucleotides both including relevant epigenetic modifications, e.g. guanine methylation, and the incorporation of others nucleic bases such thymine within the oligonucleotide sequence.

\section{Conclusions}

In this article, the electrochemical response of enzymatically treated 6-mer oligonucleotides has been studied by SWV using screen-printed graphite electrodes (SPGE). The results obtained show a clear improvement on the electrochemical response when enzymatically treated polynucleotides based on dGMP and dAMP nucleotides are used. A facile and cost effective protocol of enzymatic digestion of oligonucleotides has been designed through the use of the Nuclease S1 enzyme. 0.1 $\mathrm{M}$ acetate buffer solution $\mathrm{pH}$ 5.0, sample overnight incubation at room temperature, no need of using EDTA solutions for ceasing the enzymatic reaction and removal 
of filtration step have been considered as the most important variables for the optimization of the protocol. Enzymatically treated oligonucleotides have depicted a linear dependence between current intensity and concentration. Remarkably, the electrochemical sensitivity was clearly improved for enzymatically treated samples which may have important consequences for the development of a future electroanalytical method for DNA composition determination. This study provides a proof of principle to employ the voltammetric response of both digested and undigested polynucleotides to acquire information about composition, sequence and concentration of short DNA fragments within a solution using a low cost disposable SPGE.

\section{Acknowledgements}

This work has been also financially supported by the MINECO (Spain) through projects CTQ2013-48280-C33-R and CTQ2013-44083-P.

\section{References}

[1] B. Alberts, Molecular Biology of the Cell: Reference Edition, Taylor \& Francis, 2008.

[2] a) S. B. Baylin, J. G. Herman, J. R. Graff, P. M. Vertino, J. P. Issa, Adv. Cancer Res. 1998, 72, 141-196; b) L. Parry, A. R. Clarke, Genes Cancer 2011, 2, 618-630.

[3] J. Chen, O. Odenike, J. D. Rowley, Nat. Rev. Cancer 2010, 10, 23-36.

[4] S. A. Belinsky, K. J. Nikula, W. A. Palmisano, R. Michels, G. Saccomanno, E. Gabrielson, S. B. Baylin, J. G. Herman, P. Natl. Acad. Sci. USA 1998, 95, 11891-11896.

[5] K. V. Donkena, C. Y. F. Young, D. J. Tindall, Obstet. Gynecol. Int. 2010, 2010, 302051-302051.

[6] a) K. Patterson, L. Molloy, W. Qu, S. Clark, J. Vis. Exp. 2011, e3170; b) J. G. Herman, J. R. Graff, S. Myöhänen, B. D. Nelkin, S. B. Baylin, P. Natl. Acad. Sci. USA 1996, 93, 9821-9826.

[7] R. C. Gupta, M. V. Reddy, K. Randerath, Carcinogenesis 1982, 3, 1081-1092.

[8] a) P. G. Foiles, S. A. Akerkar, S. G. Carmella, M. Kagan, G. D. Stoner, J. H. Resau, S. S. Hecht, Chem. Res. Toxicol. 1991, 4, 364-368; b) S. Sonoki, J. Lin, S. Hisamatsu, Anal. Chim. Acta 1998, 365, 213-217; c) Y. Esaka, S. Inagaki, M. Goto, Journal of Chromatography B-Analytical Technologies in the Biomedical and Life Sciences 2003, 797, 321-329; d) S. Cortacero-Ramirez, A. Segura-Carretero, C. Cruces-Blanco, M. RomeroRomero, A. Fernandez-Gutierrez, Anal. Bioanal. Chem. 2004, 380, 831-837; e) R. Singh, P. B. Farmer, Carcinogenesis 2006, 27, 178-196.

[9] a) G. Chen, X. Han, L. Zhang, J. Ye, J. Chromatogr. A 2002, 954, 267-276; b) G. Chen, Q. Chu, L. Zhang, J. Ye, Anal. Chim. Acta 2002, 457, 225-233.
[10] a) D. L. Smith, P. J. Elving, J. Am. Chem. Soc. 1962, 84, 1412-1420; b) D. L. Smith, P. J. Elving, J. Am. Chem. Soc. 1962, 84, 2741-2747; c) E. Paleček, B. Janík, Arch. Biochem. Biophys. 1962, 98, 527-528; d) T. E. Cummings, M. A. Jensen, P. J. Elving, Bioelectroch. Bioener. 1977, 4, 425-446.

[11] A. Brotons, F. J. Vidal-Iglesias, J. Solla-Gullon, J. Iniesta, Anal. Methods 2016, 8, 702-715.

[12] A. Brotons, L. A. Mas, J. P. Metters, C. E. Banks, J. Iniesta, Analyst 2013, 138, 5239-5249.

[13] a) M. S. Goh, M. Pumera, Electroanal. 2012, 24, 11471152; b) M. S. Goh, A. Bonanni, A. Ambrosi, Z. Sofer, M. Pumera, Analyst 2011, 136, 4738-4744; c) R. J. Toh, A. Bonanni, M. Pumera, Electrochem. Commun. 2012, 22, 207-210.

[14] Q. Li, C. Batchelor-McAuley, R. G. Compton, J. Phys. Chem. B 2010, 114, 7423-7428.

[15] a) T. A. Ivandini, B. V. Sarada, T. N. Rao, A. Fujishima, Analyst 2003, 128, 924-929; b) F. Jelen, M. Tomschik, E. Palecek, J. Electroanal. Chem. 1997, 423, 141-148; c) C. Prado, G. U. Flechsig, P. Grundler, J. S. Foord, F. Marken, R. G. Compton, Analyst 2002, 127, 329-332.

[16] D. Kato, K. Goto, S.-i. Fujii, A. Takatsu, S. Hirono, O. Niwa, Anal. Chem. 2011, 83, 7595-7599.

[17] P. M. Hallam, D. K. Kampouris, R. O. Kadara, C. E. Banks, Analyst 2010, 135, 1947-1952.

[18] R. O. Kadara, N. Jenkinson, C. E. Banks, Sensor. Actuat. B-Chem. 2009, 138, 556-562.

[19] S. C. Hillier, C. G. Frost, A. T. A. Jenkins, H. T. Braven, R. W. Keay, S. E. Flower, J. M. Clarkson, Bioelectrochemistry 2004, 63, 307-310.

[20] N. A. Desai, V. Shankar, FEMS Microbiology Reviews 2003, 26, 457-491.

[21] K. Goto, D. Kato, N. Sekioka, A. Ueda, S. Hirono, O. Niwa, Anal. Biochem. 2010, 405, 59-66.

[22] A. Brotons, I. Sanjuan, C. E. Banks, F. J. Vidal-Iglesias, J. Solla-Gullón, J. Iniesta, Electroanal. 2015, 27, 27662772.

[23] I. Sanjuán, A. Brotons, N. Hernández-Ibáñez, C. W. Foster, C. E. Banks, J. Iniesta, Electrochim. Acta 2015. 
